\title{
Semi-analytical solution for dynamic behavior of a fluid-conveying pipe with different boundary conditions
}

\author{
Xu Liang ${ }^{1}$, Xing Zha ${ }^{1}$, Xue Jiang ${ }^{*}, 2$, Lizhong Wang ${ }^{1}$, Jianxing Leng ${ }^{1}$, Zeng Cao ${ }^{1}$ \\ ${ }^{1}$ : Ocean College, Zhejiang University, Hangzhou, Zhejiang 310058, China \\ 2: Dept. of Naval Architecture, Ocean and Marine Engineering, University of Strathclyde, \\ Henry Dyer Building, Glasgow, G1 5AE, UK
}

\begin{abstract}
This paper analyzes the dynamic behavior of a fluid-conveying pipe with different pipe end boundary conditions. The pipe is considered to be an Euler-Bernoulli beam, and a motion equation for the pipe is derived using Hamilton's principle. A semi-analytical method, which includes the differential quadrature method (DQM) and the Laplace transform and its inverse, is used to obtain a model for the dynamic behavior of the pipe. The use of DQM provides a solution in terms of pipe length whereas use of the Laplace transform and its inverse produce a solution in terms of time. An examination of the results of sampling pipe displacement at different numbers of sample points along the pipe length shows that the method we developed has a fast convergence rate. The frequency and critical velocity of the fluid-conveying pipe derived by DQM are exactly the same as the exact solution. The numerical results given by the model match well with the result obtained using the Galerkin method. The effect on pipe displacement of the pipe end boundary conditions is investigated, and it increases with an increase in the edge degrees of freedom. The results obtained in this paper can serve as benchmark data in further research.
\end{abstract}

Keywords: Dynamic response; Differential quadrature method; Numerical inversion of Laplace transform; Galerkin method.

\section{Introduction}

The vibration of a fluid-conveying pipe is a concern in many fields, such as marine engineering, aviation, construction machinery, chemical engineering, and oil exploration and refining. Thus improved knowledge of this phenomenon is widely applicable. A major risk for a free spanning pipeline is the failure caused by fatigue associated with various loads. The relatively large amplitudes of the oscillations of a free spanning pipe in vertical direction, caused by the external load, can lead to fatigue damage to the pipeline. Being able to accurately model the dynamic behavior of a fluid-conveying pipe is therefore important for theory development and has practical engineering significance.

The dynamic behavior of a fluid-conveying pipe became one of the hot topics since Brillouin (Paidoussis, 1998) first observed flow-induced vibration. Long (1955) first studied the vibration characteristics of a fluid-conveying pipe by experimental method, and observed 
that the natural frequency of the pipe decreases with the increase of the internal fluid velocity. Benjamin (1961a, 1961b) discussed the dynamic behaviors of a cantilevered fluid-conveying pipe, and found that flutter instability occurs in the pipe. Gregory and Paidoussis (1966a, 1966b) confirmed that the first unstable mode of the cantilevered fluid-conveying pipe is flutter instability instead of divergent instability through theoretical and experimental method, and investigated the effects of the mass ratio, damping and stiffness on critical velocity of instability. Chen (1970) investigated the forced vibration of a fluid-conveying pipe. Paidoussis and Issid (1974) gave the linear governing equation of the fluid-conveying pipe considering a variety of factors, and studied the vibration instability of the pipe under different boundary conditions. Paidoussis (1987) published a review article on the instability of cylindrical structures caused by fluid, which described the linear vibration of the pipe in detail, and pointed out two instability phenomena, namely flutter instability and buckling instability. More details on this subject can be referred in some monographs and reviews (Paidoussis, 1987; Paidoussis and Li, 1993). A variety of calculation methods have been proposed for the dynamic behaviors of the fluid-conveying pipe due to its wide application. Ibrahim $(2010,2011)$ and Li et al. (2015) have systematically summarized the progress of research into fluid-conveying pipe vibrations. Dai et al. (2014) studied a flexible pipe conveying fluctuating flows and analyzed the principal parametric resonances during lock-in for each of the first two modes using the direct perturbation method of multiple scales (MMS). He et al. (2017) enumerated the characteristics of vortex-induced vibrations-in a pipe for the first two locked-in models under quasi-static displacement conditions using the Galerkin method. Li and Yang (2017) applied He's variational iteration method (HVIM) to obtain the critical flow velocity and frequency of a fluid-conveying pipe under various boundary conditions. Zhai et al. (2011) analyzed the dynamic response of a Timoshenko pipeline (beam) under random excitation using the pseudo excitation method and the complex mode superposition method. Mohammadimehr and Mehrabi (2017) investigated the stability and free vibration of double-bonded micro-composite-sandwich cylindrical shells under magneto-thermo-mechanical loadings using the generalized differential quadrature method (GDQM). Kuiper and Metrikine (2005) used power series expansion and D-decomposition to study the stability of a vertically suspended free-hanging riser, and explained instability at small velocities of convection. Lin and Qiao (2008) used the differential quadrature method (DQM) to model the dynamical behavior of a fluid-conveying curved pipe subjected to motion-limiting constraints and harmonic excitation. Li and Yang (2014) obtained exact solutions for forced vibration of a fluid-conveying pipe using the Green function, and derived the natural frequencies of the fluid-conveying pipe. Chatzopoulou et al. (2016) investigated the vibration and instability of cyclically-loaded steel pipes during deep water reeling installation using the finite element method (FEM), and examined the effects of the modulus and the damping factor of the linear viscoelastic Winkler foundation and the fluid velocity on the resonance frequencies. Ni et al. (2011) used the differential transformation method (DTM) to analyze the vibration problem of a fluid-conveying pipe with several typical boundary conditions, and obtained natural frequencies and critical flow velocities for pipes. Hashemian and Mohareb (2016) suggested the finite difference model to analyze the sandwich pipes with thick cores subjected to internal and external hydrostatic pressure. Gu et al. (2016) modeled the dynamic response of a fluid-conveying pipe using a generalized integral transform 
technique (GITT), and analyzed the effect of aspect ratio on deflection and natural frequencies. Yazdi (2013) discussed the nonlinear vibration of doubly curved cross-ply shells using the homotopy perturbation method (HPM).

Comprehensive reviews of the calculation methods for vibration of fluid-conveying pipes are given in the above literature. However, a semi-analytical solution for the dynamic behavior of a fluid-conveying pipe has not yet been proposed. We propose a more accurate semi-analytical methodology that incorporates the differential quadrature method (DQM) and the inverse Laplace transform to investigate the dynamic behavior of a fluid-conveying pipe.

\section{Method}

\subsection{Laplace transform and its numerical inversion}

The Laplace transform is a linear transform which is widely used in structural dynamics. It can convert partial differential equations into ordinary differential equations or transform ordinary differential equations into algebraic equations (Liang et al., 2014). The Laplace transform and its inverse have been successfully applied to investigate the dynamic response of the structures subject to external load (Khalili et al., 2009; Lou and Klosner, 1973). Suppose $f(\mathrm{t})$ is a real-valued function of time $t$, which defined in the real domain $[0,+\infty)$, the Laplace transform and its inversion are defined by:

$$
\begin{gathered}
\tilde{f}(s)=L[f(t)]=\int_{0}^{+\infty} f(t) e^{-s t} d t \\
f(t)=L^{-1}[\tilde{f}(s)]=\frac{1}{2 \pi i} \int_{\alpha-i \infty}^{\alpha+i \infty} \tilde{f}(s) e^{s t} d s
\end{gathered}
$$

where $L$ denotes the Laplace transform; $L^{-1}$ denotes the inverse Laplace transform; $s$ is a complex number in the Laplace domain; $s_{i}(\mathrm{i}=1,2,3, \ldots)$ are the singularities of $\tilde{f}(s) ; \operatorname{Re}$ denotes the real part of the complex number. Let $s=\alpha+i \omega$, where $\alpha$ and $\omega$ are real numbers; the convergence condition of (2) can be stated as $\alpha>\alpha_{0}=\max \left[\operatorname{Re}\left(s_{i}\right)\right]$.

Durbin (1974) proposed that for the interval (0, T/2), the Laplace transform can be computed to any desired accuracy by the following formula (Liang et al., 2014):

$$
f(t) \cong \frac{2 e^{\beta t}}{T}\left\{\frac{\tilde{f}(\beta)}{2}+\sum_{n=1}^{N} \operatorname{Re}[\tilde{f}(\beta+n \pi i / T)] \cos (n \pi t / T)\right\}
$$

where $\beta=5 / T, \mathrm{~T}=5 \times T_{d}, T_{d}$ is the observing period in the time domain, and $N$ is a large integer.

\subsection{Differential quadrature method (DQM)}

The differential quadrature method was first introduced into the field of structural dynamics by Bert and Malik (1996). In order to convert the derivative term with respect to the $x$ coordinate to polynomials, the differential quadrature method (DQM) is employed to discretize the fundamental equations. Considering a continuous function $f(x)$, the $n$th order partial derivative with respect to $x$ at a given point $x=x_{i}$ can be approximated by a linear weighted sum of function values at all the sample points in the domain of $x$, that is (Liang et al., 2015):

$$
\frac{\partial^{n} f\left(x_{i}\right)}{\partial x^{n}}=\sum_{i=1}^{M} A_{i j}^{(n)} f\left(x_{i}\right)(i, j=1,2, \ldots M)
$$


where $\mathrm{M}$ is the number of sample points, and $A_{\mathrm{ij}}^{(\mathrm{n})}$ are the weighting coefficients of the $n$th order derivative defined by:

$$
\begin{gathered}
A_{i j}^{(1)}=\frac{\prod_{k=1, k \neq i}^{M}\left(x_{i}-x_{k}\right)}{\left(x_{i}-x_{j}\right) \prod_{k=1, k \neq j}^{M}\left(x_{j}-x_{k}\right)} \\
A_{i j}^{(k)}=k\left(A_{i j}^{(k-1)} A_{i j}^{1}-\frac{A_{i j}^{(k-1)}}{x_{i}-x_{j}}\right)(k=1,2, \ldots M-1)
\end{gathered}
$$

where $i, j=1,2, \ldots M$, but $i \neq j$, and the $A_{\mathrm{ii}}^{(\mathrm{n})}$ are defined as:

$$
A_{i i}^{(k)}=-\sum_{j=1, j \neq i}^{M} A_{i j}^{(k)}(i=1,2, \ldots M, k=1,2, \ldots M-1)
$$

\section{Problem description}

\subsection{Governing equations}

In this paper, we consider a linear elastic pipe of length $L$, which internally conveys an incompressible fluid with a velocity $v$ and which is subjected to harmonic load $q$. The geometry and the Cartesian coordinate system are shown in Fig. 1, in which the origin of the $(x, y, z)$ framework is considered to be located in space at the left end of the pipe (Chen, 1970; Dai et al., 2014).

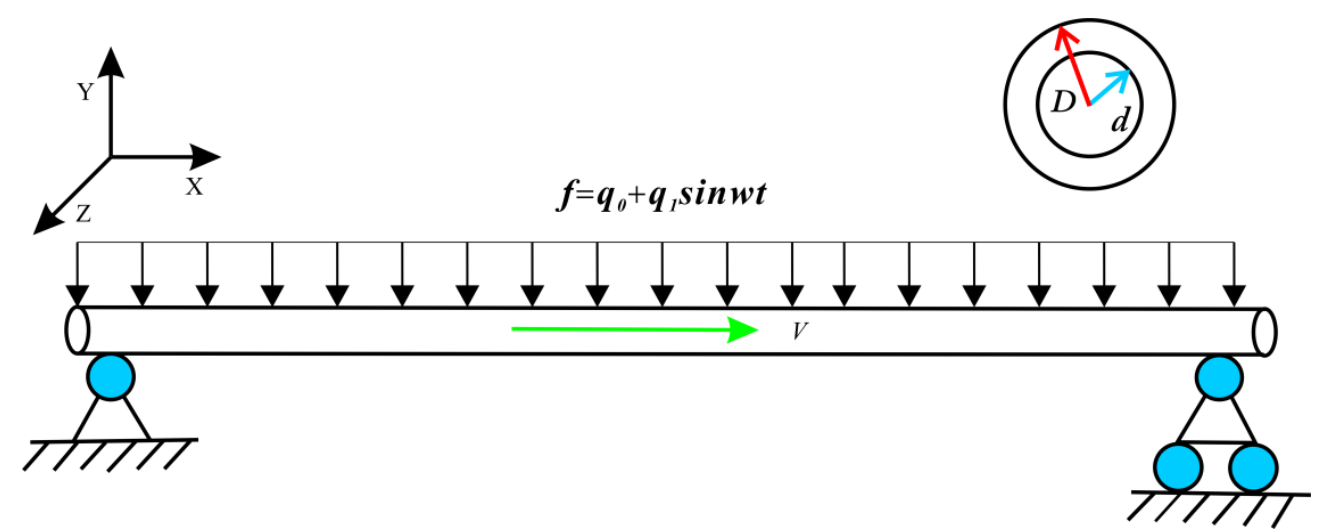

Fig. 1 The geometry of a pipe subjected to harmonic load.

The motion of the pipe in the vertical ( $y$-axis) direction has been considered. In this paper, the following assumptions are made: (1) the fluid within the pipe (internal fluid) is incompressible and has a constant velocity; (2) the cross section of the elastic pipe is uniform, and the effects of shear, torsion, and rotational inertia can be neglected; and (3) the motion of the pipe is considered to be only in the vertical ( $y$-axis) direction.

Adopting a small deformation assumption and treating the pipe as an Euler-Bernoulli beam, the equation for the motion of the pipe can be derived using Hamilton's principle. The kinetic energy of the system includes the kinetic energy of the pipe and the kinetic energy of the internal fluid, and is described by the equation: 


$$
T=\frac{1}{2} \int_{0}^{l} m_{p}\left(\frac{\partial w}{\partial t}\right)^{2} d x+\frac{1}{2} \int_{0}^{l} m_{f}\left(\left(v \frac{\partial w}{\partial x}+\frac{\partial w}{\partial t}\right)^{2}+v^{2}\right) d x
$$

where $w$ refers to the deflection in the vertical (y-axis) direction, $m_{p}$ and $m_{f}$ respectively denote the mass per unit length of the pipe and the internal fluid. The deformation energy of the system is

$$
U=\frac{1}{2} \int_{0}^{l} E I\left(\frac{\partial^{2} w}{\partial x^{2}}\right)^{2} d x
$$

where $E I$ is the flexural rigidity of the pipe. The work done by non-conservative forces is derived from the harmonic load, which can be written as:

$$
\delta W_{n c}=\int_{0}^{l} f(x, t) \delta w d x=\int_{0}^{l}\left(q_{0}+q_{1} \sin \omega t\right) \delta w d x
$$

where $f(x, t)$ is the harmonic load.

According to Hamilton's principle, the functional of the system can be written as

$$
\int_{t_{1}}^{t_{2}} \delta(T-U) d t+\int_{t_{1}}^{t_{2}} \delta W_{n c} d t=0
$$

where $T$ is the total kinetic energy of the system; $U$ is the deformation energy of the system; $W_{\text {nc }}$ denotes the work of the non-conservative force. By substituting Eqs. (8)-(10) into Eq. (11), the following expression is obtained:

$$
E I \frac{\partial^{4} w}{\partial x^{4}}+m_{f} v^{2} \frac{\partial^{2} w}{\partial x^{2}}+2 m_{f} v \frac{\partial^{2} w}{\partial x \partial t}+\left(m_{p}+m_{f}\right) \frac{\partial^{2} w}{\partial t^{2}}=f(x, t)
$$

where $w(x, t)$ is the displacement of the pipe in the longitudinal direction (y-axis); $x$ and $t$ are the axial coordinate and time, respectively.

Introducing the following non-dimensional quantities:

$$
\begin{gathered}
\eta=\frac{w}{l}, \xi=\frac{x}{l}, \beta=\frac{m_{f}}{m_{p}+m_{f}}, \tau=\frac{t}{l^{2}}\left(\frac{E I}{m_{p}+m_{f}}\right)^{1 / 2}, u=v l\left(\frac{m_{f}}{E I}\right)^{1 / 2} \\
\bar{q}_{0}=\frac{q_{0} l^{3}}{E I}, \bar{q}_{1}=\frac{q_{1} l^{3}}{E I}, \bar{\omega}=\omega l^{2}\left(\frac{m_{p}+m_{f}}{E I}\right)^{1 / 2}
\end{gathered}
$$

Eq. (12) can be rewritten as a dimensionless form

$$
\frac{\partial^{4} \eta}{\partial \xi^{4}}+u^{2} \frac{\partial^{2} \eta}{\partial \xi^{2}}+2 \sqrt{\beta} u \frac{\partial^{2} \eta}{\partial \xi \partial \tau}+\frac{\partial^{2} \eta}{\partial \tau^{2}}=\bar{q}_{0}+\bar{q}_{1} \sin \bar{\omega} \tau
$$

\subsection{The boundary conditions at the edges}

The fluid-conveying pipe is usually considered to be simply supported or clamped at the ends of the pipe $(\xi=0$ and $\xi=1)$. Four combinations of pipe end boundary conditions are considered here: simply supported-simply supported, clamped-clamped, simply supported-clamped and clamped-free, which can be symbolized as S-S, C-C, S-C and C-F, respectively. The boundary conditions at the edges can be expressed as follows.

Simply supported $(\xi=0)-$ Simply supported $(\xi=1)$, S-S

at $\xi=0, \quad \eta=0$ and $\partial^{2} \eta / \partial \xi^{2}=0$, 
at $\xi=1, \quad \eta=0$ and $\partial^{2} \eta / \partial \xi^{2}=0$.

Clamped $(\xi=0)-$ Clamped $(\xi=1), \mathrm{C}-\mathrm{C}$

at $\xi=0, \quad \eta=0$ and $\partial \eta / \partial \xi=0$,

at $\xi=1, \quad \eta=0$ and $\partial \eta / \partial \xi=0$.

Simply supported $(\xi=0)-$ Clamped $(\xi=1), \mathrm{S}-\mathrm{C}$

at $\xi=0, \quad \eta=0$ and $\partial^{2} \eta / \partial \xi^{2}=0$,

at $\xi=1, \quad \eta=0$ and $\partial \eta / \partial \xi=0$.

Clamped $(\xi=0)-$ Free $(\xi=1), \mathrm{C}-\mathrm{F}$

at $\xi=0, \quad \eta=0$ and $\partial \eta / \partial \xi=0$,

at $\xi=1, \quad \partial^{2} \eta / \partial \xi^{2}=0$ and $\partial^{3} \eta / \partial \xi^{3}=0$.

\section{Solution procedure}

\subsection{Natural frequency calculation}

To obtain the natural frequencies of fluid-conveying pipe, external load is ignored here. The fundamental equation can be derived as follows:

$$
\frac{\partial^{4} \eta}{\partial \xi^{4}}+u^{2} \frac{\partial^{2} \eta}{\partial \xi^{2}}+2 \sqrt{\beta} u \frac{\partial^{2} \eta}{\partial \xi \partial \tau}+\frac{\partial^{2} \eta}{\partial \tau^{2}}=0
$$

The solution of Eq. (23) can be expressed as

$$
\eta=\bar{\eta} e^{\Omega \tau}
$$

where $\Omega$ denotes eigenvalue of system.

Take the fluid-conveying pipe with boundary condition $S-S$ as an example. By substituting Eq. (24) into Eq. (23) and Eqs. (15) - (16), and then applying DQM on the new equations, the following equations can be derived:

$$
\begin{gathered}
\sum_{j=1}^{N} A_{i j}^{(4)} \bar{\eta}_{j}+u^{2} \sum_{j=1}^{N} A_{i j}^{(2)} \bar{\eta}_{j}+2 \sqrt{\beta} u \Omega \sum_{j=1}^{N} A_{i j}^{(1)} \bar{\eta}_{j}+\Omega^{2} \bar{\eta}_{i}=0 \quad(i=3,4, \cdots N-2) \\
\bar{\eta}_{1}=0, \quad \sum_{j=1}^{N} A_{1 j}^{(2)} \bar{\eta}_{j}=0 \\
\bar{\eta}_{N}=0, \quad \sum_{j=1}^{N} A_{N j}^{(2)} \bar{\eta}_{j}=0
\end{gathered}
$$

Eq. (25) can be rewritten in the following matrix form:

$$
\begin{aligned}
& {\left[\begin{array}{ll}
\mathbf{A}_{11} & \mathbf{A}_{12} \\
\mathbf{A}_{21} & \mathbf{A}_{22}
\end{array}\right]\left\{\begin{array}{l}
\overline{\boldsymbol{\eta}}_{1} \\
\overline{\boldsymbol{\eta}}_{2}
\end{array}\right\}+\Omega\left[\begin{array}{cc}
\mathbf{0} & \mathbf{0} \\
\mathbf{B}_{21} & \mathbf{B}_{22}
\end{array}\right]\left\{\begin{array}{l}
\overline{\boldsymbol{\eta}}_{1} \\
\overline{\boldsymbol{\eta}}_{2}
\end{array}\right\}+\Omega^{2}\left[\begin{array}{ll}
\mathbf{0} & \mathbf{0} \\
\mathbf{0} & \mathbf{I}
\end{array}\right]\left\{\begin{array}{l}
\overline{\boldsymbol{\eta}}_{1} \\
\overline{\boldsymbol{\eta}}_{2}
\end{array}\right\}=\left\{\begin{array}{l}
\mathbf{0} \\
\mathbf{0}
\end{array}\right\},} \\
& \overline{\boldsymbol{\eta}}_{\mathbf{1}}=\left\{\begin{array}{llll}
\bar{\eta}_{1} & \bar{\eta}_{2} & \bar{\eta}_{N-1} & \bar{\eta}_{N}
\end{array}\right\}, \quad \overline{\boldsymbol{\eta}}_{\mathbf{2}}=\left\{\begin{array}{llll}
\bar{\eta}_{3} & \bar{\eta}_{4} & \cdots & \bar{\eta}_{N-2}
\end{array}\right\}
\end{aligned}
$$


The simplified equations can be obtained by eliminating the dimensionless displacement vector $\overline{\boldsymbol{\eta}}_{1}$ as follows:

$$
\left(\Omega^{2} \mathbf{M}+\Omega \mathbf{C}+\mathbf{K}\right)\left\{\overline{\boldsymbol{\eta}}_{\mathbf{2}}\right\}=\mathbf{0}
$$

where $\mathbf{I}$ denotes unit matrix, and the specific forms of matrix $\mathbf{C}$ and $\mathbf{K}$ are defined by

$$
\mathbf{C}=-\mathbf{B}_{21} \mathbf{A}_{11}^{-1} \mathbf{A}_{12}+\mathbf{B}_{22}, \quad \mathbf{K}=-\mathbf{A}_{21} \mathbf{A}_{11}^{-1} \mathbf{A}_{12}+\mathbf{A}_{22}
$$

The solution of Eq. (27) can be converted to solve eigenvalues of the following matrix:

$$
\mathbf{E}=\left[\begin{array}{cc}
\mathbf{0} & \mathbf{I} \\
-\mathbf{M}^{-1} \mathbf{K} & -\mathbf{M}^{-1} \mathbf{C}
\end{array}\right]
$$

The eigenvalues $\boldsymbol{\Omega}$ of matrix $\mathbf{E}$ is a series of complex numbers, the imaginary part of eigenvalues $\operatorname{Im}(\Omega)$ are the natural frequencies of the fluid-conveying pipe.

\subsection{Dynamic response calculation}

The fluid-conveying pipe was assumed to be in a quiescent state at the initial time. The initial conditions are:

$$
\eta(\xi, 0)=0, \quad \partial \eta(\xi, 0) / \partial \tau=0
$$

By employing the Laplace transform and DQM, the governing equation can be rewritten in terms of the Laplace function:

$$
\begin{gathered}
\sum_{j=1}^{N} A_{i j}^{(4)} \tilde{\eta}\left(\xi_{j}, s\right)+u^{2} \sum_{j=1}^{N} A_{i j}^{(2)} \tilde{\eta}\left(\xi_{j}, s\right)+2 \sqrt{\beta} u s \sum_{j=1}^{N} A_{i j}^{(1)} \tilde{\eta}\left(\xi_{j}, s\right) \\
+s^{2} \tilde{\eta}\left(\xi_{i}, s\right)=\tilde{f}
\end{gathered}
$$

Eq. (31) can be rewritten in matrix form:

$$
\left\{\begin{array}{c}
\tilde{f}_{1} \\
\tilde{f}_{2} \\
\tilde{f}_{3} \\
\vdots \\
\tilde{f}_{N-2} \\
\tilde{f}_{N-1} \\
\tilde{f}_{N}
\end{array}\right\}=\left[\begin{array}{ccccccc}
H_{11} & H_{12} & H_{13} & \cdots & H_{1, N-2} & H_{1, N-1} & H_{1 N} \\
H_{21} & H_{22} & H_{23} & \cdots & H_{2, N-2} & H_{2, N-1} & H_{2 N} \\
H_{31} & H_{32} & H_{33} & \cdots & H_{3, N-2} & H_{3, N-1} & H_{3 N} \\
\vdots & \vdots & \vdots & \ddots & \vdots & \vdots & \vdots \\
H_{N-2,1} & H_{N-2,2} & H_{N-2,3} & \cdots & H_{N-2, N-2} & H_{N-2, N-1} & H_{N-2, N} \\
H_{N-1,1} & H_{N-1,2} & H_{N-1,3} & \cdots & H_{N-1, N-2} & H_{N-1, N-1} & H_{N-1, N} \\
H_{N 1} & H_{N 2} & H_{N 3} & \cdots & H_{N, N-2} & H_{N, N-1} & H_{N N}
\end{array}\right]\left\{\begin{array}{c}
\tilde{\eta}\left(\xi_{1}, s\right) \\
\tilde{\eta}\left(\xi_{2}, s\right) \\
\tilde{\eta}\left(\xi_{3}, s\right) \\
\vdots \\
\tilde{\eta}\left(\xi_{N-2}, s\right) \\
\tilde{\eta}\left(\xi_{N-1}, s\right) \\
\tilde{\eta}\left(\xi_{N}, s\right)
\end{array}\right\}
$$

where $\tilde{\eta}\left(\xi_{i}, s\right)$ is the displacement of node $i$ in the Laplace domain and $f_{i}$ is the force acting on node $i$, which can described by:

$$
\tilde{f}_{i}=\frac{\bar{q}_{0}}{s}+\bar{q}_{1} \frac{\bar{\omega}}{\bar{\omega}^{2}+s^{2}}, \quad i=1,2, \ldots, N
$$

$H_{i j}$ denotes the coefficient matrix of the governing equation, which can be expressed as:

$$
H_{i j}=A_{i j}^{(4)}+u^{2} A_{i j}^{(2)}+2 \sqrt{\beta} u s A_{i j}^{(1)}
$$

where $i, j=1,2, \ldots, \mathrm{N}$, when $i \neq j$, and the $H_{i i}$ are defined as

$$
H_{i i}=A_{i i}^{(4)}+u^{2} A_{i i}^{(2)}+2 \sqrt{\beta} u s A_{i i}^{(1)}+s^{2}
$$

Several methods can be used to incorporate the boundary conditions (Jang et al., 1989; 
Shu and Du, 1997; Wang and Gu, 2015; Wang et al., 1999), such as the $\delta$ approach, equation substitution, or SBCGE. Considering both the accuracy of results and convenience of use, the equation substitution method is used to deal with the boundary conditions herein. Taking the fluid-conveying pipe with boundary conditions $S-S$ as an example, Eq. (15) and Eq. (16) become, by the use of the Laplace transform and DQM, respectively:

$$
\begin{aligned}
& \xi=0, \quad \tilde{\eta}\left(\xi_{1}, s\right)=0 \text { and } \sum_{j=1}^{N} A_{1 j}^{(2)} \tilde{\eta}\left(\xi_{j}, s\right)=0 \\
& \xi=1, \quad \tilde{\eta}\left(\xi_{N}, s\right)=0 \text { and } \sum_{j=1}^{N} A_{N j}^{(2)} \tilde{\eta}\left(\xi_{j}, s\right)=0 .
\end{aligned}
$$

By substituting Eq. (36) and Eq. (37) into Eq. (32), the following equations are obtained:

$$
\left\{\begin{array}{c}
0 \\
0 \\
\tilde{f}_{3} \\
\vdots \\
\tilde{f}_{N-2} \\
0 \\
0
\end{array}\right\}=\left[\begin{array}{ccccccc}
1 & 0 & 0 & \cdots & 0 & 0 & 0 \\
0 & A_{12}^{(2)} & A_{13}^{(2)} & \cdots & A_{1, N-2}^{(2)} & A_{1, N-1}^{(2)} & 0 \\
0 & H_{32} & H_{33} & \cdots & H_{3, N-2} & H_{3, N-1} & 0 \\
\vdots & \vdots & \vdots & \ddots & \vdots & \vdots & \vdots \\
0 & H_{N-2,2} & H_{N-2,3} & \cdots & H_{N-2, N-2} & H_{N-2, N-1} & 0 \\
0 & A_{N 2}^{(2)} & A_{N 3}^{(2)} & \cdots & A_{N, N-2}^{(2)} & A_{N, N-1}^{(2)} & 0 \\
0 & 0 & 0 & \cdots & 0 & 0 & 1
\end{array}\right]\left\{\begin{array}{c}
\tilde{\eta}\left(\xi_{1}, s\right) \\
\tilde{\eta}\left(\xi_{2}, s\right) \\
\tilde{\eta}\left(\xi_{3}, s\right) \\
\vdots \\
\tilde{\eta}\left(\xi_{N-2}, s\right) \\
\tilde{\eta}\left(\xi_{N-1}, s\right) \\
\tilde{\eta}\left(\xi_{N}, s\right)
\end{array}\right\}
$$

After finding the nontrivial solution of Eq. (38), one can obtain the solution in terms of time through Eq. (3).

\section{Numerical results}

Mathematica software was used to create a package that performed the preceding computations. Then we took the following steps to evaluate the model. Firstly, we estimated the convergence and validity of the results when pipe displacement observations at the pipe midpoint for different numbers of sample points $(\mathrm{N})$ along the pipe length $(x$-axis). Secondly, we obtained the frequency and critical velocity of the fluid-conveying pipe. Thirdly, we compared our results with those obtained using other methods to show the correctness and accuracy of our method. Fourthly, we investigated the effects of various boundary conditions on pipe displacement. Lastly, we evaluated the effects of geometric parameters on pipe displacement. It is noted that the used parameters are considered as the following form:

$$
E=210 \mathrm{GN} / \mathrm{m}^{2}, L=10 \mathrm{~m}, D=0.25 \mathrm{~m}, d=0.125 \mathrm{~m}, \rho_{p}=8700 \mathrm{~kg} / \mathrm{m}^{3}, \rho_{f}=870 \mathrm{~kg} / \mathrm{m}^{3} \text {. }
$$

\subsection{Convergence and validity}

To demonstrate the accuracy of this method, when compared with other methods, we analyzed the convergence of pipe dimensionless displacement values observed at different numbers of sample points $\mathrm{N}$ along the pipe length ( $x$-axis). Taking the $\mathrm{S}-\mathrm{S}$ at the ends as an example, the convergence of values obtained when displacement was observed at different numbers of sample points $\mathrm{N}$ along the pipe length ( $x$-axis) was analyzed. A series of sample points $\mathrm{N}=7,9,13$ and 15 is used in this section. Fig. 2 shows the time histories of the fluid-conveying pipe at a given displacement position $(\mathrm{x}=0.5 \mathrm{~L})$ along the $x$-axis. As is shown in Figure 2, time histories of the pipe coincide when $\mathrm{N}$ is greater than 9, which shows 
that the developed method quickly converges when there are more than 9 sample points.

The validity of the developed method is checked again in this section. The results obtained by the developed method are compared with the results generated by Fourier series expansion technique and Galerkin method. A simply supported fluid-conveying pipe is considered and the number of sample points is selected as 11 for convergence. The time histories of pipe dimensionless displacement at the midpoint $(x=\mathrm{L} / 2)$ are plotted in Fig. 3 . It is obvious that the results obtained by the developed method agree well with the results generated by Fourier series expansion technique and Galerkin method.

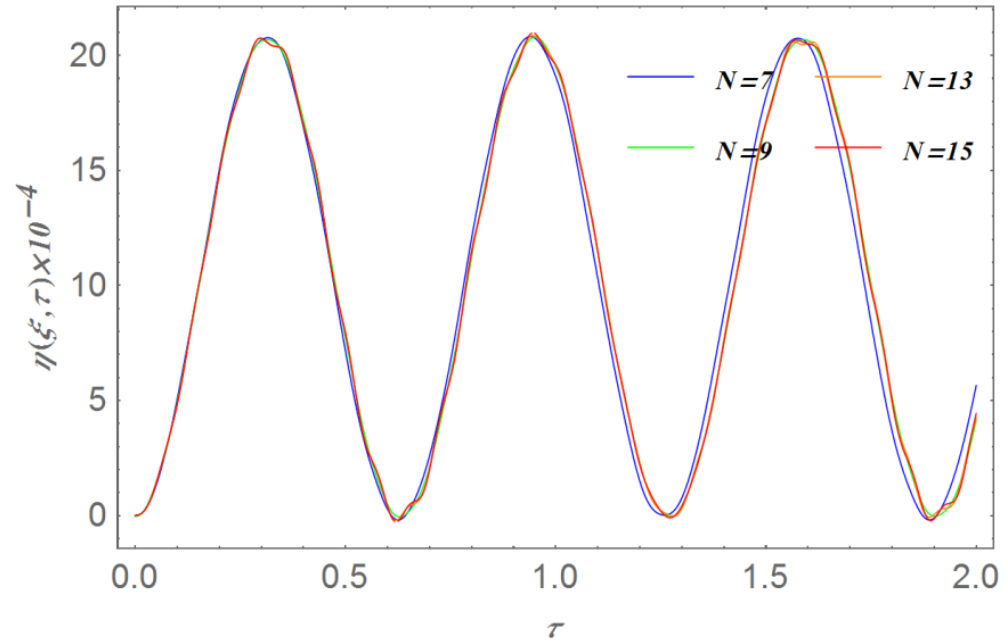

Fig. 2 The $\mathrm{S}-\mathrm{S}$ time histories of pipe dimensionless displacement at the midpoint $(x=\mathrm{L} / 2)$ for four different numbers of sample points along the $x$-direction.

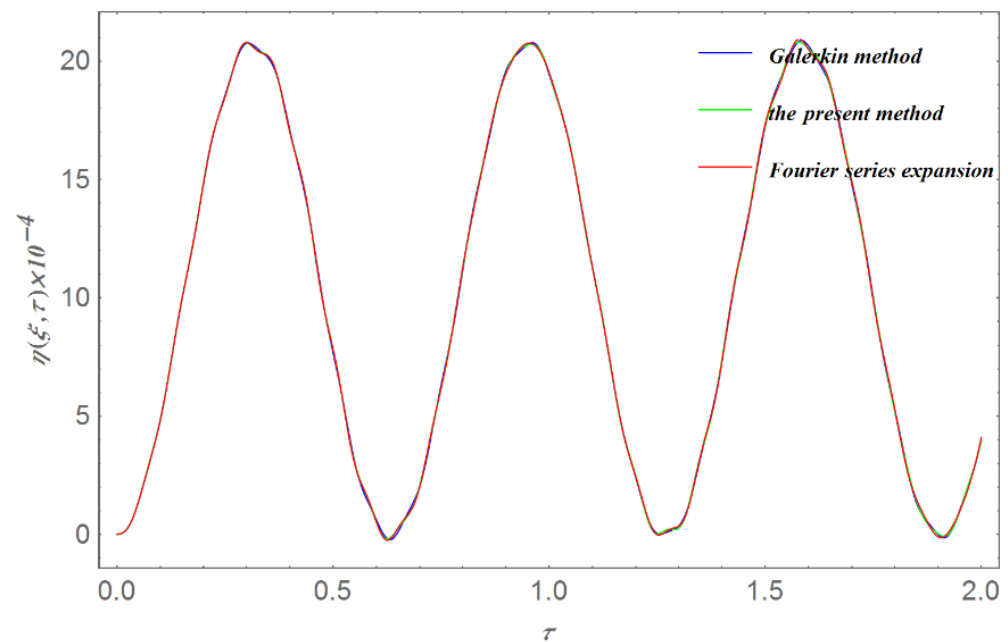

Fig. 3 The S-S time histories of pipe dimensionless displacement at the midpoint $(x=\mathrm{L} / 2)$ using the developed method, Galerkin method and Fourier series expansion technique.

\subsection{Frequency and critical velocity}

Table1 shows the first four frequencies of the pipe with different boundary conditions for $u=0$ produced by a series of sample points, and shows that results are exactly the same as the exact results from $\mathrm{Ni}$ et al. (2011) and Thomson (1988). It is noted that the number of sample point is chosen to be $\mathrm{N}=30$ for higher accuracy.

In the case of $u \neq 0$, Figs. 4-7 represent the natural frequencies of fluid-conveying pipe with different boundary conditions. The first three dimensionless natural frequencies of the S-S fluid-conveying pipe with $0 \leq \mathrm{u} \leq 9$ are plotted in Fig. 4 . The first mode appears divergence 
instability when the critical velocity of the pipe is $u=3.14$, and coupled-mode flutter instability appears when the critical velocity is $\mathrm{u}=6.31$. Fig. 5 shows the first three dimensionless natural frequencies of the $\mathrm{C}-\mathrm{C}$ fluid-conveying pipe with $0 \leq \mathrm{u} \leq 10$. The critical velocity of the pipe is $u=6.28$ and 9.30 , and corresponds to divergence instability in the first mode and couple-mode flutter instability.

Table 1 Natural frequency of the pipe with different boundary conditions for $u=0$.

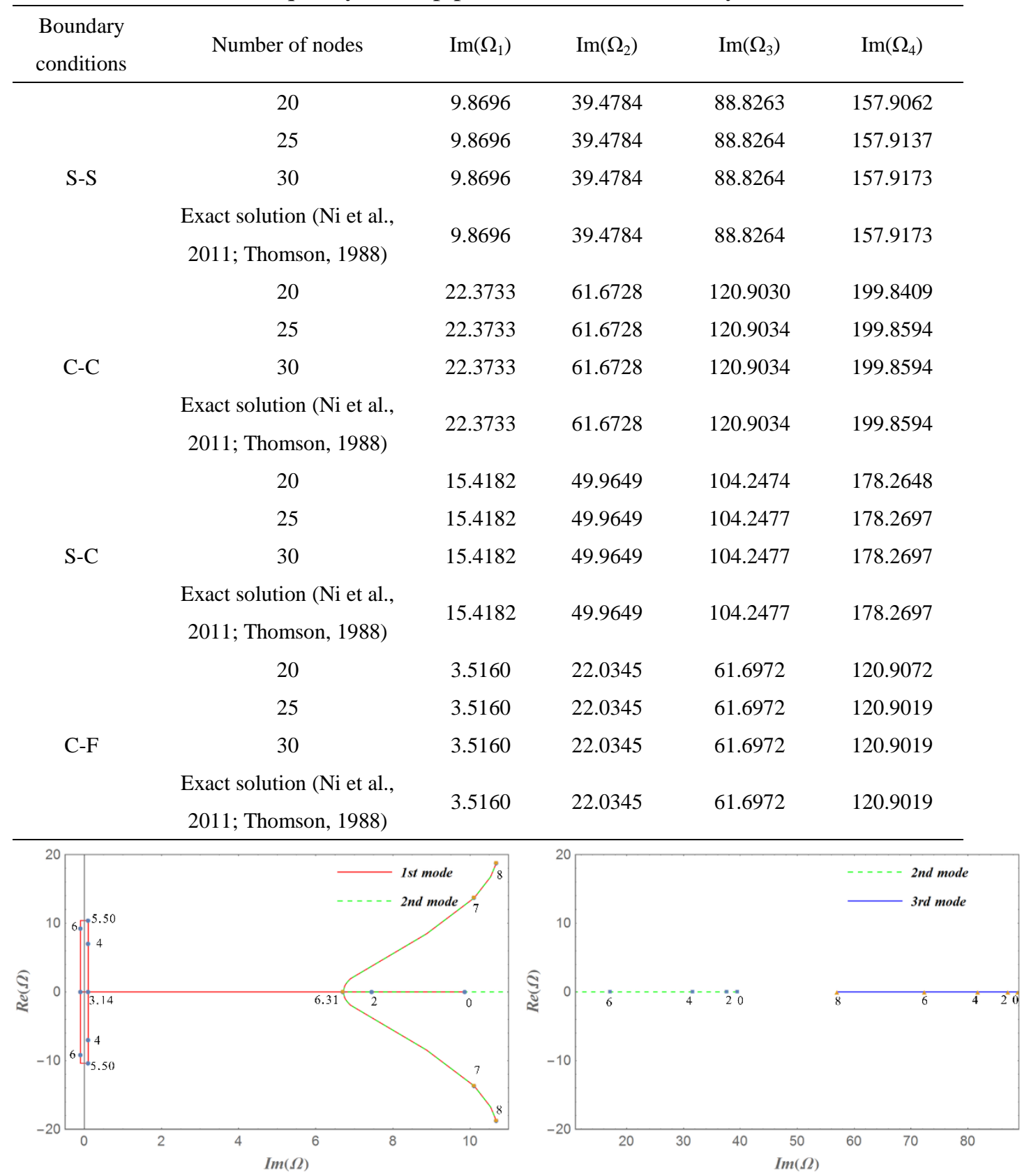

Fig. 4 The first three dimensionless natural frequencies of the $S-S$ fluid-conveying pipe with $\beta=0.5$. 

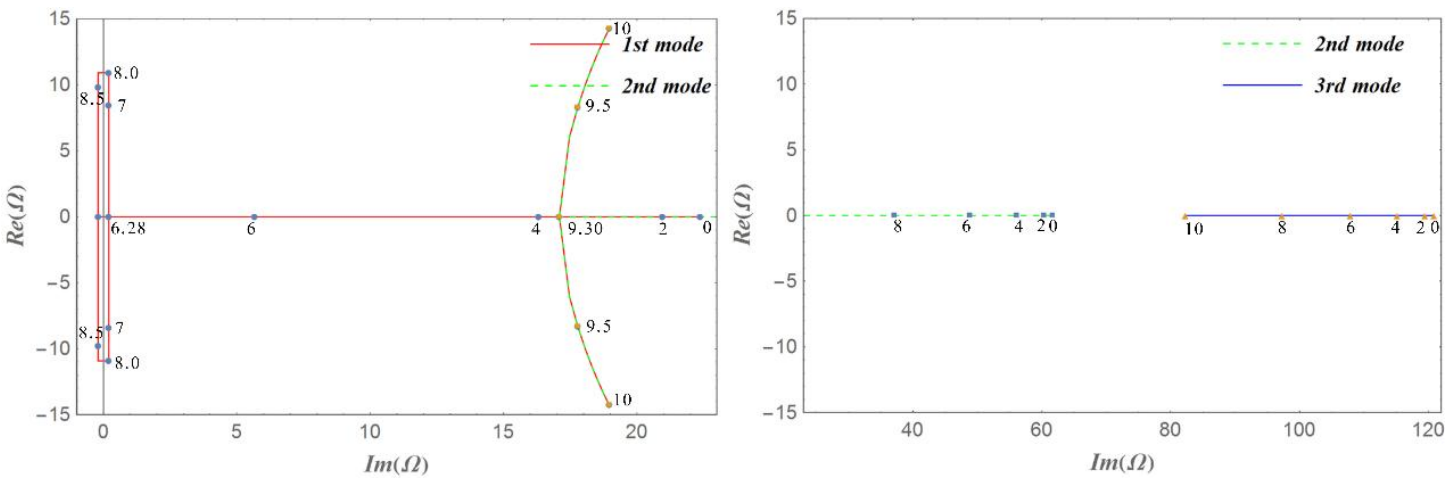

Fig. 5 The first three dimensionless natural frequencies of the $\mathrm{C}-\mathrm{C}$ fluid-conveying pipe with $\beta=0.5$.

Fig. 6 presents the first three dimensionless natural frequency of the $\mathrm{S}-\mathrm{C}$ fluid-conveying pipe with $0 \leq \mathrm{u} \leq 10$. It is obvious that the first mode appears divergence instability when fluid velocity $\mathrm{u}=4.49$, and coupled-mode flutter instability appears when fluid velocity reaches to $\mathrm{u}=7.78$. The first three dimensionless natural frequencies of the $\mathrm{C}-\mathrm{F}$ fluid-conveying pipe with $0 \leq \mathrm{u} \leq 10$ are depicted in Fig. 7. The critical velocity of the pipe is $u=9.33$, and the third mode appears flutter instability. The specific critical velocities under different boundary conditions are listed in Table 2 .
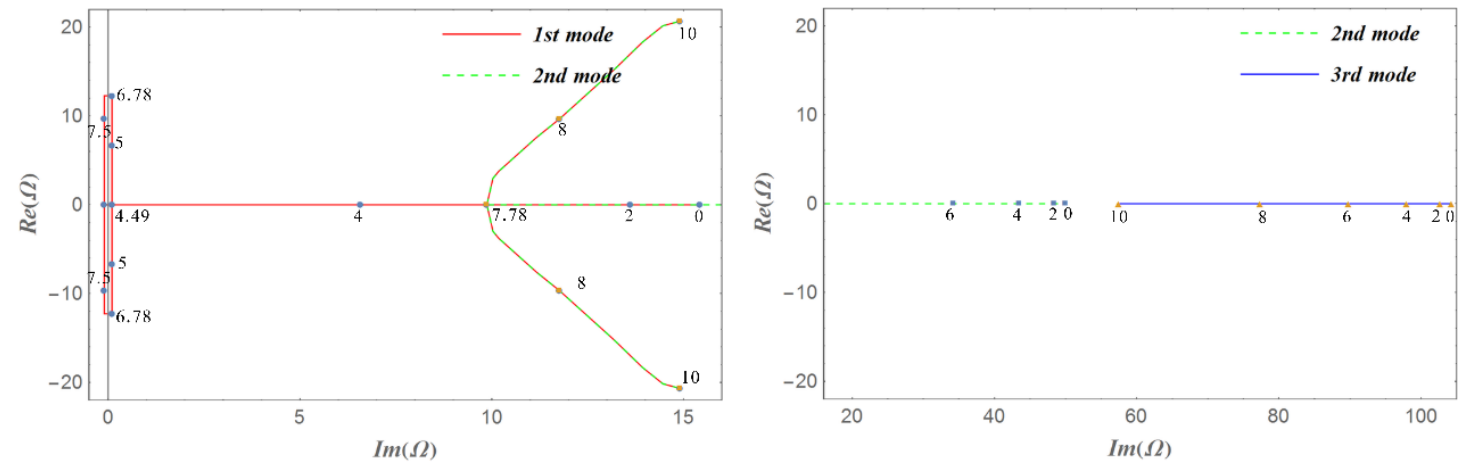

Fig. 6 The first three dimensionless natural frequencies of the $S-C$ fluid-conveying pipe with $\beta=0.5$.

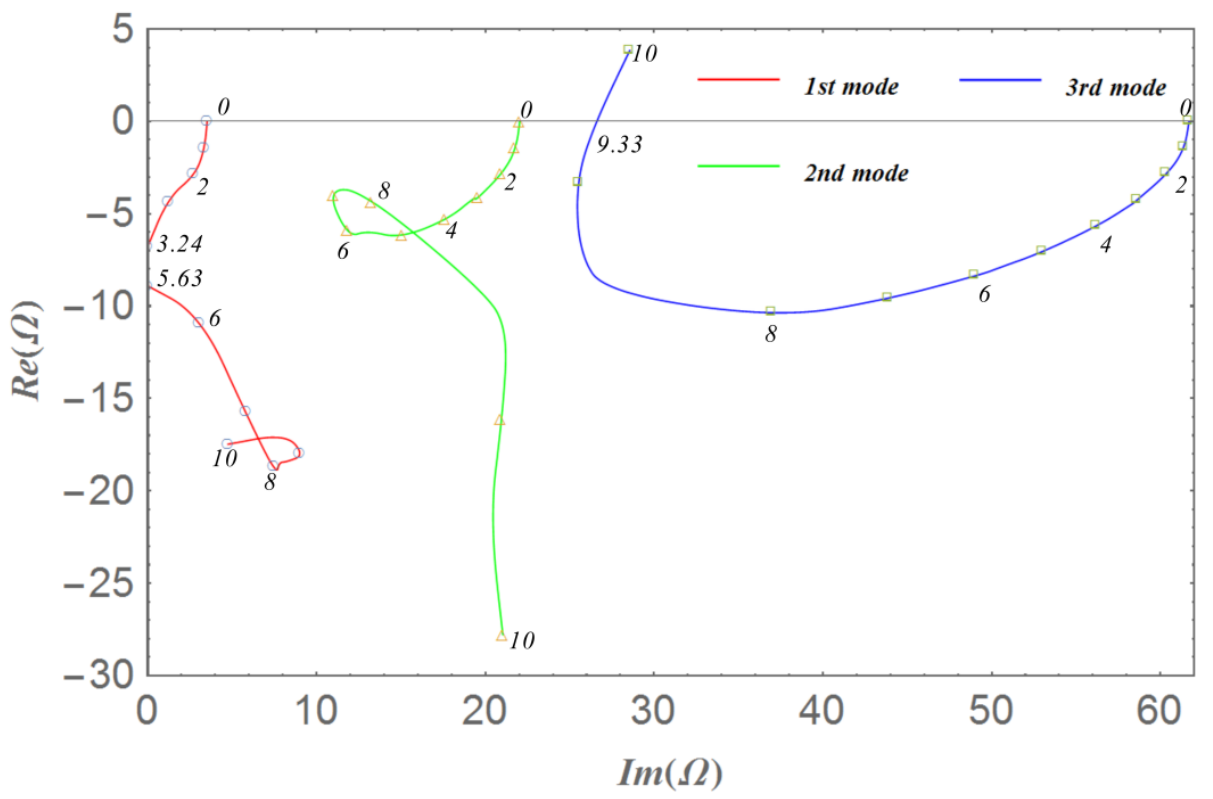


Fig. 7 The first three dimensionless natural frequencies of the C-F fluid-conveying pipe with $\beta=0.5$.

Table 2 Critical velocities of the fluid-conveying pipe under different boundary conditions, $\beta=0.5$.

\begin{tabular}{cccccc}
\hline S-S & $\mathrm{u}$ & Instability form & $\mathrm{C}-\mathrm{C}$ & $\mathrm{u}$ & Instability form \\
\hline 1st mode & 3.14 & divergence & 1st mode & 6.28 & divergence \\
1st and 2nd combined & 6.31 & coupled-mode flutter & 1st and 2nd combined & 9.30 & coupled-mode flutter \\
\hline S-C & $\mathrm{u}$ & Instability form & $\mathrm{C}-\mathrm{F}$ & $\mathrm{u}$ & Instability form \\
\hline 1st mode & 4.49 & divergence & 3rd mode & 9.33 & flutter \\
1st and 2nd combined & 7.78 & coupled-mode flutter & - & - & - \\
\hline
\end{tabular}

\subsection{Comparison to other methods}

To demonstrate the increased accuracy of the method used in this paper, our semi-analytical result is compared with results obtained by using the Galerkin method, the Newmark- $\beta$ method and the Wilson- $\theta$ method. The fluid-conveying pipe has S-S boundary conditions at the ends, and 15 sample points was used along the length of the pipe. The four-order precision Galerkin method is taken for higher accuracy. In order to ensure the convergence of results, the following parameter values in Newmark- $\beta$ method and the Wilson- $\theta$ method were used: $\gamma \geq 0.5, \beta \geq(0.5+\gamma)^{2} / 4$ (Newmark- $\beta$ ); $\theta=1.366$ (Wilson- $\theta$ ).

The time histories of the pipe dimensionless displacement at the midpoint $(x=\mathrm{L} / 2)$ are plotted in Fig. 8. The result produced by the Galerkin method completely coincides with the result by using the method developed method in this paper. However, there is a slight difference when the results of our method are compared to those of the Newmark- $\beta$ method and the Wilson- $\theta$ method. The reason is that the Wilson- $\theta$ method has a higher algorithmic damping and the Newmark- $\beta$ will both decrease the cycle frequency of the time history (Bathe and Wilson, 2010). It is clear that the method developed in this paper has greater accuracy and is a simpler calculation than the other methods. 


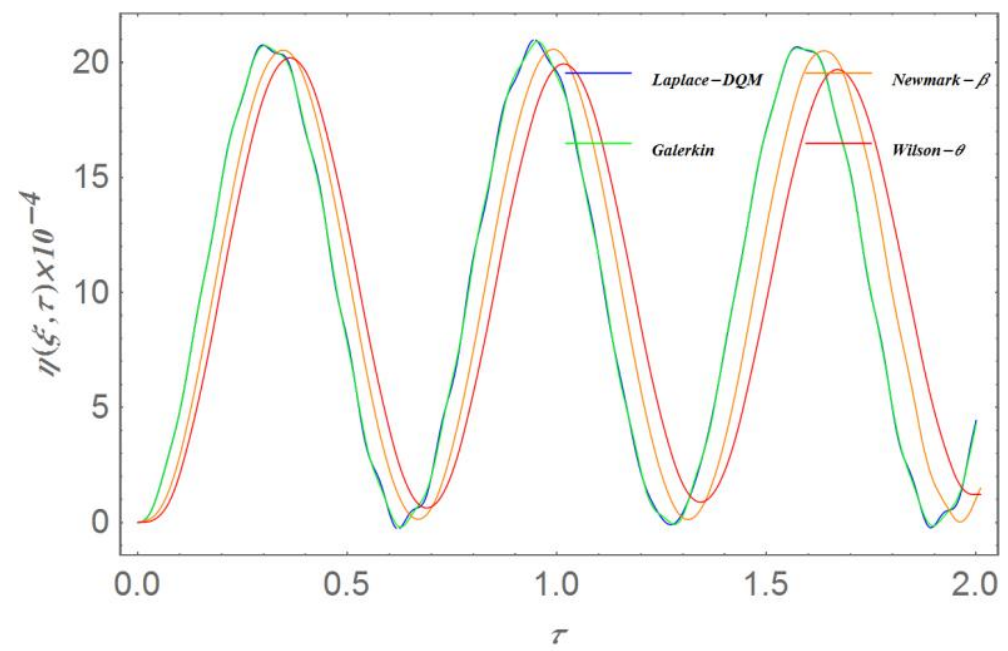

Fig. 8 The $\mathrm{S}-\mathrm{S}$ time histories of the pipe dimensionless displacement at the midpoint $(x=\mathrm{L} / 2)$ using the different methods.

\subsection{The effects of boundary conditions at the ends}

The effects of the boundary conditions at the ends of the pipe on pipe behavior were investigated. The differences in extent of pipe displacement for each of the four sets of boundary conditions were considered: $\mathrm{S}-\mathrm{S}, \mathrm{S}-\mathrm{C}, \mathrm{C}-\mathrm{C}, \mathrm{C}-\mathrm{F}$. It is noted that the length of the pipe is chosen to be $\mathrm{L}=7 \mathrm{~m}$, and the sample points is taken as $\mathrm{N}=15$ for accuracy and convergence. The time histories of the fluid-conveying pipe at the midpoint ( $x=\mathrm{L} / 2)$ under different boundary conditions at the ends are given in Fig. 9. The peak displacement of the fluid-conveying pipe with end boundary conditions $\mathrm{C}-\mathrm{F}$ is largest in all boundary conditions, and the pipe with end boundary conditions $\mathrm{C}-\mathrm{C}$ is smallest. It is obvious that the peak displacement of the pipe increases with an increase in the total number of degrees of freedom at the ends.

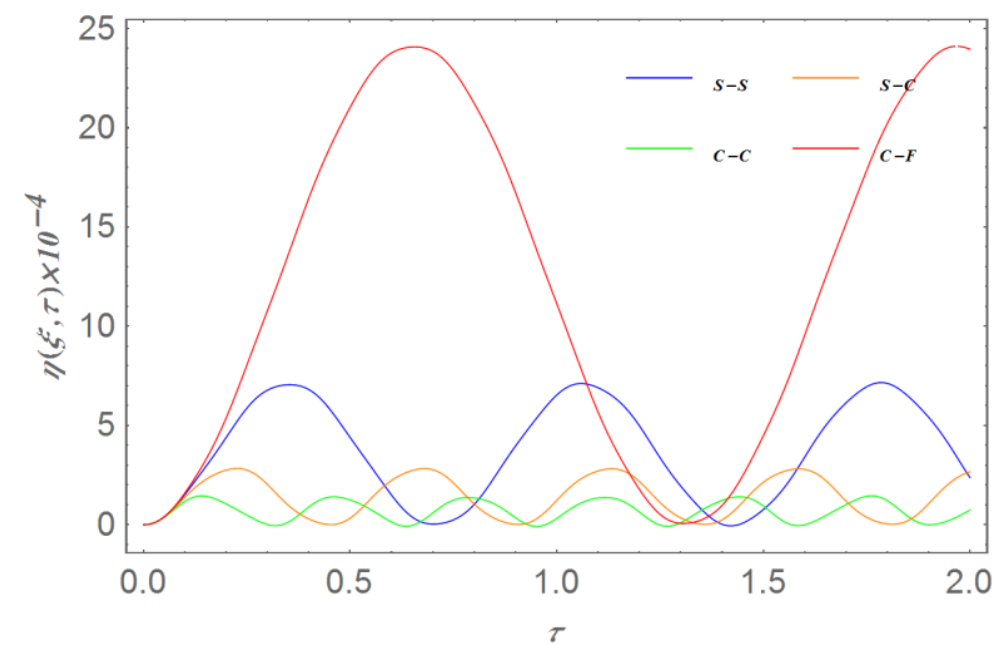

Fig. 9 Time histories of pipe dimensionless displacement under four different sets of end boundary conditions.

\subsection{The effects of geometric parameters}

The effects of geometric parameters on pipe displacement are evaluated in this section. Two series of geometric parameters are considered here (the first series: $\mathrm{L} / \mathrm{R}=60,65,70$ and 75 ; the second series: $\mathrm{h} / \mathrm{R}=0.2,0.3,0.4$ and 0.5 ). The boundary condition of the fluid-conveying pipe is supposed to be simply supported at both ends and the number of 
sample points is selected as 11 for accuracy. The time histories of pipe displacement with different geometric parameters are presented in Fig. 10 and Fig. 11. It is shown that the peak displacement of the fluid-conveying pipe increases with the increase of length to radius ratio and the decrease of thickness to radius ratio.

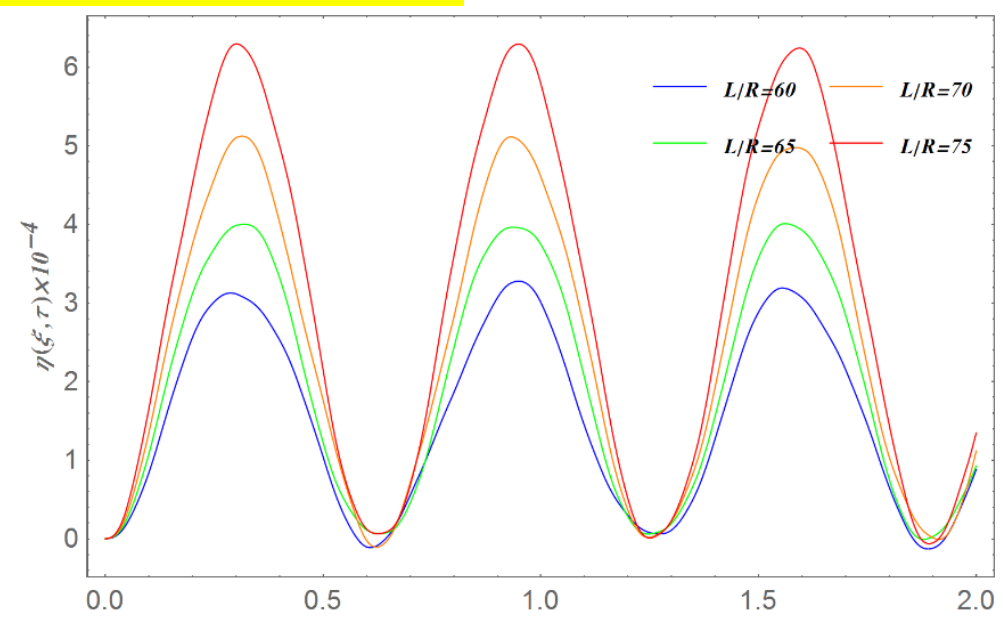

Fig. 10 The S-S time histories of the pipe dimensionless displacement at the midpoint $(\mathrm{x}=$ $\mathrm{L} / 2$ ) with different length to radius ratios.

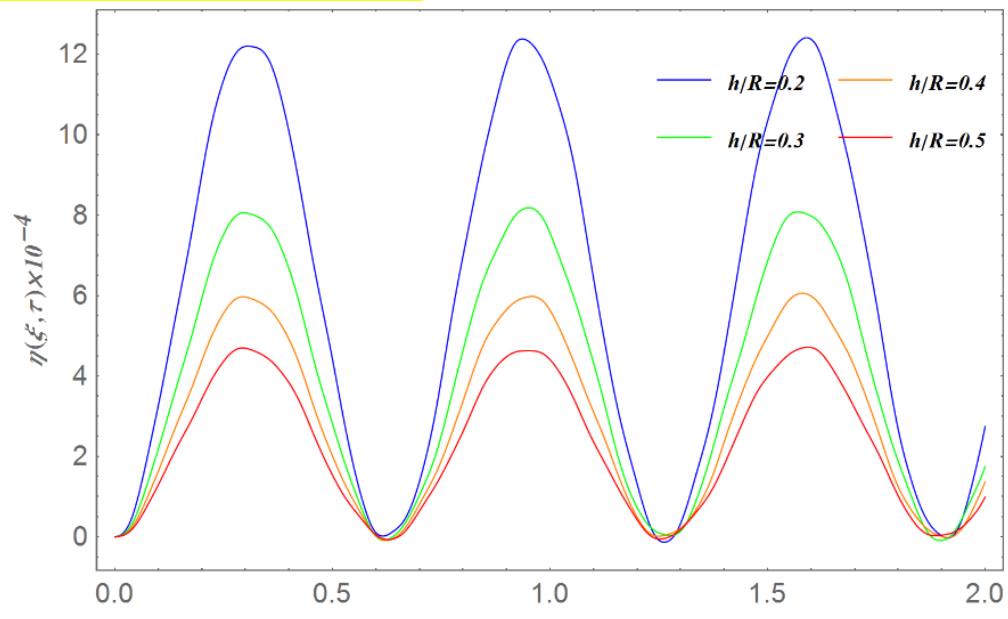

Fig. 11 The S-S time histories of the pipe dimensionless displacement at the midpoint $(\mathrm{x}=$ $\mathrm{L} / 2$ ) with different thickness to radius ratios.

\section{Conclusions}

The dynamic characteristics of a fluid-conveying pipe are much studied. The complex modal analytical method and the Galerkin method are the most common methods for analyzing and modeling the dynamic behavior of the pipe. We have developed a semi-analytical methodology which is simple and accurate in order to model the dynamic response of a fluid-conveying pipe. The methodology incorporates the differential quadrature method (DQM), the Laplace transform and its inverse.

To demonstrate the improved accuracy of the model we have developed, a comparison of our results with those from other methods is carried out. It shows that the results generated by the Galerkin method are the same as those of our model. Examination of the convergence of the results by taking different numbers of sample observations along the pipe length shows 
that our method has a fast convergence rate. The frequency and critical velocity are obtained using DQM, and numerical results show a good agreement with the exact solution. The effects on pipe displacement of the boundary conditions and geometric parameters are analyzed. Numerical results show that the peak displacement of the pipe with C-F boundary condition is larger than that other boundary conditions and the displacement increases with an increase in the edge degrees of freedom, and the peak displacement of the pipe increases as length to radius ratio increases or thickness to radius ratio decreases.

\section{Acknowledgment}

The authors gratefully acknowledge the financial support provided by and the National Natural Science Foundation of China (Nos.51679214, 51409228), the Fundamental Research Funds for the Central Universities (2018QNA4047),

\section{References}

Bathe, K.J., Wilson, E.L., 2010. Solution methods for eigenvalue problems in structural mechanics. International Journal for Numerical Methods in Engineering 6 (2), 213-226.

Benjamin, T.B., 1961a. Dynamics of a system of articulated pipes conveying fluid. I. Theory. Proceedings of the Royal Society of London 261 (1307), 487-499.

Benjamin, T.B., 1961b. Dynamics of a system of articulated pipes conveying fluid. II. Experiments. Proceedings of the Royal Society of London 261 (1307), 487-499.

Bert, C.W., Malik, M., 1996. Free vibration analysis of thin cylindrical shells by the differential quadrature method. Vitis -Geilweilerhof- 118 (1), 1-12.

Chatzopoulou, G., Karamanos, S.A., Varelis, G.E., 2016. Finite element analysis of cyclically-loaded steel pipes during deep water reeling installation. Ocean Engineering 124, 113-124.

Chen, S.S., 1970. Forced vibration of a cantilevered tube conveying fluid. Journal of the Acoustical Society of America 48 (3B), 771-781.

Dai, H.L., Wang, L., Qian, Q., Ni, Q., 2014. Vortex-induced vibrations of pipes conveying pulsating fluid. Ocean Engineering 77 (2), 12-22.

Durbin, F., 1974. Numerical inversion of Laplace Transforms: An efficient improvement to Dubner and Abate's method. Computer Journal 17 (4), 371-376.

Gregory, R.W., Paidoussis, M.P., 1966a. Unstable oscillation of tubular cantilevers conveying fluid. I. Theory. Proceedings of the Royal Society of London 293 (1435), 512-527.

Gregory, R.W., Paidoussis, M.P., 1966b. Unstable oscillation of tubular cantilevers conveying fluid. II. Experiments. Proceedings of the Royal Society of London 293 (1435), 528-542.

Gu, J., Ma, T., Duan, M., 2016. Effect of aspect ratio on the dynamic response of a fluid-conveying pipe using the Timoshenko beam model. Ocean Engineering 114, 185-191. Hashemian, R., Mohareb, M., 2016. Finite difference model for the buckling analysis of sandwich pipes under external pressure. Ocean Engineering 122, 172-185.

He, F., Dai, H., Huang, Z., Wang, L., 2017. Nonlinear dynamics of a fluid-conveying pipe under the combined action of cross-flow and top-end excitations. Applied Ocean Research 62, 199-209.

Ibrahim, R.A., 2010. Overview of mechanics of pipes conveying fluids-Part I: Fundamental Studies. Journal of Pressure Vessel Technology 132 (3). 
Ibrahim, R.A., 2011. Mechanics of pipes conveying fluids-Part II: Applications and Fluidelastic Problems. Journal of Pressure Vessel Technology 133 (2), 140-144.

Jang, S.K., Bert, C.W., Striz, A.G., 1989. Application of differential quadrature to static analysis of structural components. International Journal for Numerical Methods in Engineering 28 (3), 561-577.

Khalili, S.M.R., Azarafza, R., Davar, A., 2009. Transient dynamic response of initially stressed composite circular cylindrical shells under radial impulse load. Composite Structures 89 (2), 275-284.

Kuiper, G.L., Metrikine, A.V., 2005. Dynamic stability of a submerged, free-hanging riser conveying fluid. Journal of Sound \& Vibration 280 (3-5), 1051-1065.

Li, S., Karney, B.W., Liu, G., 2015. FSI research in pipeline systems - A review of the literature. Journal of Fluids \& Structures 57, 277-297.

Li, Y.D., Yang, Y.R., 2014. Forced vibration of pipe conveying fluid by the Green function method. Archive of Applied Mechanics 84 (12), 1811-1823.

Li, Y.D., Yang, Y.R., 2017. Vibration analysis of conveying fluid pipe via He's variational iteration method. Applied Mathematical Modelling 43, 409-420.

Liang, X., Kou, H.L., Wang, L., Palmer, A.C., Wang, Z., Liu, G., 2015. Three-dimensional transient analysis of functionally graded material annular sector plate under various boundary conditions. Composite Structures 132, 584-596.

Liang, X., Wu, Z., Wang, L., Liu, G., Wang, Z., Zhang, W., 2014. Semianalytical three-dimensional solutions for the transient response of functionally graded material rectangular plates. Journal of Engineering Mechanics 141 (9).

Lin, W., Qiao, N., 2008. Nonlinear dynamics of a fluid-conveying curved pipe subjected to motion-limiting constraints and a harmonic excitation. Journal of Fluids \& Structures 24 (1), 96-110.

Long, R.H., 1955. Experimental and theoretical study of transverse vibration of a tube containing flowing fluid. Journal of Applied Mechanics 22.

Lou, Y.K., Klosner, J.M., 1973. Transient response of a point-excited submerged spherical shell. Journal of Applied Mechanics 40 (4), 1078-1084.

Mohammadimehr, M., Mehrabi, M., 2017. Stability and free vibration analyses of double-bonded micro composite sandwich cylindrical shells conveying fluid flow. Applied Mathematical Modelling 47, 685-709.

Ni, Q., Zhang, Z.L., Wang, L., 2011. Application of the differential transformation method to vibration analysis of pipes conveying fluid. Applied Mathematics \& Computation 217 (16), 7028-7038.

Paidoussis, M.P., 1987. Flow-induced instabilities of cylindrical structures. Applied Mechanics Reviews 40 (2), 163.5

Paidoussis, M.P., 1998. Fluid-Structure Interactions: Slender Structures and Axial Flow. Academic Press, London.

Paidoussis, M.P., Issid, N.T., 1974. Dynamic stability of pipes conveying fluid. Journal of Sound \& Vibration 33 (3), 267-294.

Paidoussis, M.P., Li, G.X., 1993. Pipes conveying fluid: a model dynamical problem. Journal of Fluids \& Structures 7 (2), 137-204.

Shu, C., Du, H., 1997. Implementation of clamped and simply supported boundary conditions 
in the GDQ free vibration analysis of beams and plates. International Journal of Solids \& Structures 34 (7), 819-835.

Thomson, W.T., 1988. Theory of Vibration with Applications. Unwin Hyman Ltd, London.

Wang, X., Gu, H., 2015. Static analysis of frame structures by the differentail quadrature element method. International Journal for Numerical Methods in Engineering 40 (4), 759-772.

Wang, Y., Liu, R., Wang, X., 1999. Free vibration analysis of truncated conical shells by the differential quadrature method. Journal of Sound \& Vibration 224 (2), 387-394.

Yazdi, A.A., 2013. Applicability of homotopy perturbation method to study the nonlinear vibration of doubly curved cross-ply shells. Composite Structures 96 (96), 526-531.

Zhai, H.B., Wu, Z.Y., Liu, Y.S., Yue, Z.F., 2011. Dynamic response of pipeline conveying fluid to random excitation. Nuclear Engineering \& Design 241 (8), 2744-2749. 


\section{List of Tables}

Table 1 Natural frequency of the pipe with different boundary conditions for $u=0$.

Table 2 Critical velocities of the fluid-conveying pipe under different boundary conditions, $\beta=0.5$. 
Table 1 Natural frequency of the pipe with different boundary conditions for $u=0$.

\begin{tabular}{|c|c|c|c|c|c|}
\hline $\begin{array}{l}\text { Boundary } \\
\text { conditions }\end{array}$ & Number of nodes & $\operatorname{Im}\left(\Omega_{1}\right)$ & $\operatorname{Im}\left(\Omega_{2}\right)$ & $\operatorname{Im}\left(\Omega_{3}\right)$ & $\operatorname{Im}\left(\Omega_{4}\right)$ \\
\hline \multirow{4}{*}{ S-S } & 20 & 9.8696 & 39.4784 & 88.8263 & 157.9062 \\
\hline & 25 & 9.8696 & 39.4784 & 88.8264 & 157.9137 \\
\hline & 30 & 9.8696 & 39.4784 & 88.8264 & 157.9173 \\
\hline & $\begin{array}{c}\text { Exact solution (Ni et al., } \\
\text { 2011; Thomson, 1988) }\end{array}$ & 9.8696 & 39.4784 & 88.8264 & 157.9173 \\
\hline \multirow{4}{*}{$\mathrm{C}-\mathrm{C}$} & 20 & 22.3733 & 61.6728 & 120.9030 & 199.8409 \\
\hline & 25 & 22.3733 & 61.6728 & 120.9034 & 199.8594 \\
\hline & 30 & 22.3733 & 61.6728 & 120.9034 & 199.8594 \\
\hline & $\begin{array}{c}\text { Exact solution (Ni et al., } \\
\text { 2011; Thomson, 1988) }\end{array}$ & 22.3733 & 61.6728 & 120.9034 & 199.8594 \\
\hline \multirow{4}{*}{$\mathrm{S}-\mathrm{C}$} & 20 & 15.4182 & 49.9649 & 104.2474 & 178.2648 \\
\hline & 25 & 15.4182 & 49.9649 & 104.2477 & 178.2697 \\
\hline & 30 & 15.4182 & 49.9649 & 104.2477 & 178.2697 \\
\hline & $\begin{array}{l}\text { Exact solution (Ni et al., } \\
\text { 2011; Thomson, 1988) }\end{array}$ & 15.4182 & 49.9649 & 104.2477 & 178.2697 \\
\hline \multirow{4}{*}{$\mathrm{C}-\mathrm{F}$} & 20 & 3.5160 & 22.0345 & 61.6972 & 120.9072 \\
\hline & 25 & 3.5160 & 22.0345 & 61.6972 & 120.9019 \\
\hline & 30 & 3.5160 & 22.0345 & 61.6972 & 120.9019 \\
\hline & $\begin{array}{c}\text { Exact solution (Ni et al., } \\
\text { 2011; Thomson, 1988) }\end{array}$ & 3.5160 & 22.0345 & 61.6972 & 120.9019 \\
\hline
\end{tabular}


Table 2 Critical velocities of the fluid-conveying pipe under different boundary conditions, $\beta=0.5$.

\begin{tabular}{cccccc}
\hline S-S & $\mathrm{u}$ & Instability form & $\mathrm{C}-\mathrm{C}$ & $\mathrm{u}$ & Instability form \\
\hline 1st mode & 3.14 & divergence & 1st mode & 6.28 & divergence \\
1st and 2nd combined & 6.31 & coupled-mode flutter & 1st and 2nd combined & 9.30 & coupled-mode flutter \\
\hline S-C & $\mathrm{u}$ & Instability form & C-F & $\mathrm{u}$ & Instability form \\
\hline 1st mode & 4.49 & divergence & 3rd mode & 9.33 & flutter \\
1st and 2nd combined & 7.78 & coupled-mode flutter & - & - & - \\
\hline
\end{tabular}




\section{List of Figures}

Fig. 1 The geometry of a pipe subjected to the effect of harmonic load.

Fig. 2 The S-S time histories of pipe dimensionless displacement at the midpoint $(x=\mathrm{L} / 2)$ for four different numbers of sample points along the $x$-direction.

Fig. 3 The S-S time histories of pipe dimensionless displacement at the midpoint $(x=\mathrm{L} / 2)$ using the developed method, Galerkin method and Fourier series expansion technique.

Fig. 4 The first three dimensionless natural frequencies of the S-S fluid-conveying pipe with $\beta=0.5$.

Fig. 5 The first three dimensionless natural frequencies of the $\mathrm{C}-\mathrm{C}$ fluid-conveying pipe with $\beta=0.5$.

Fig. 6 The first three dimensionless natural frequencies of the $\mathrm{S}-\mathrm{C}$ fluid-conveying pipe with $\beta=0.5$.

Fig. 7 The first three dimensionless natural frequencies of the $\mathrm{C}-\mathrm{F}$ fluid-conveying pipe with $\beta=0.5$.

Fig. 8 The $\mathrm{S}-\mathrm{S}$ time histories of the pipe dimensionless displacement at the midpoint $(x=\mathrm{L} / 2)$ using the different methods.

Fig. 9 Time histories of pipe dimensionless displacement under four different sets of end boundary conditions.

Fig. 10 The S-S time histories of the pipe dimensionless displacement at the midpoint $(x=\mathrm{L} / 2)$ with different length to radius ratios.

Fig. 11 The $\mathrm{S}-\mathrm{S}$ time histories of the pipe dimensionless displacement at the midpoint $(x=\mathrm{L} / 2)$ with different thickness to radius ratios. 


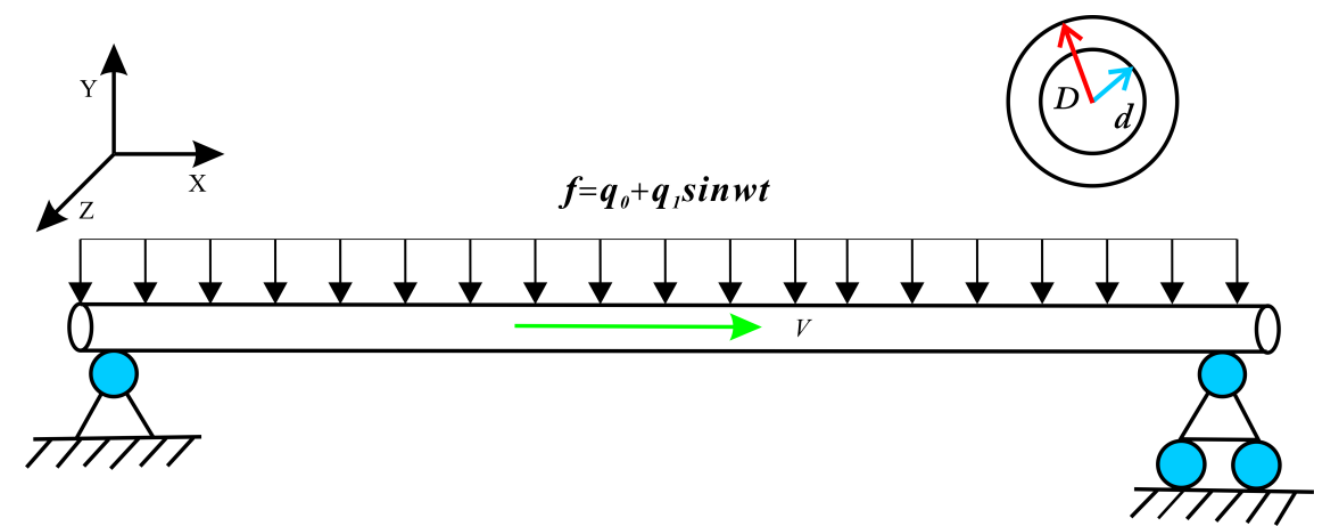

Fig. 1 The geometry of a pipe subjected to the effect of harmonic load. 


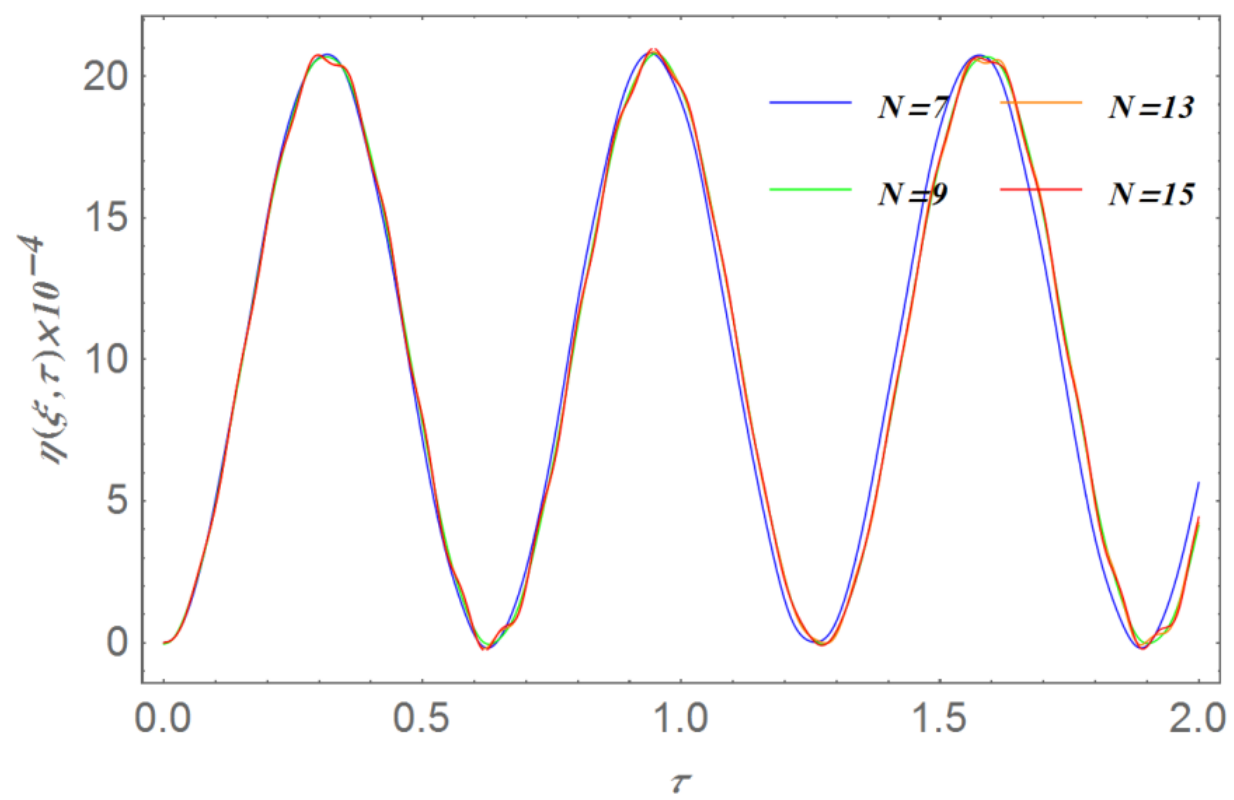

Fig. 2 The S-S time histories of pipe dimensionless displacement at the midpoint ( $x=\mathrm{L} / 2)$ for four different numbers of sample points along the $x$-direction. 


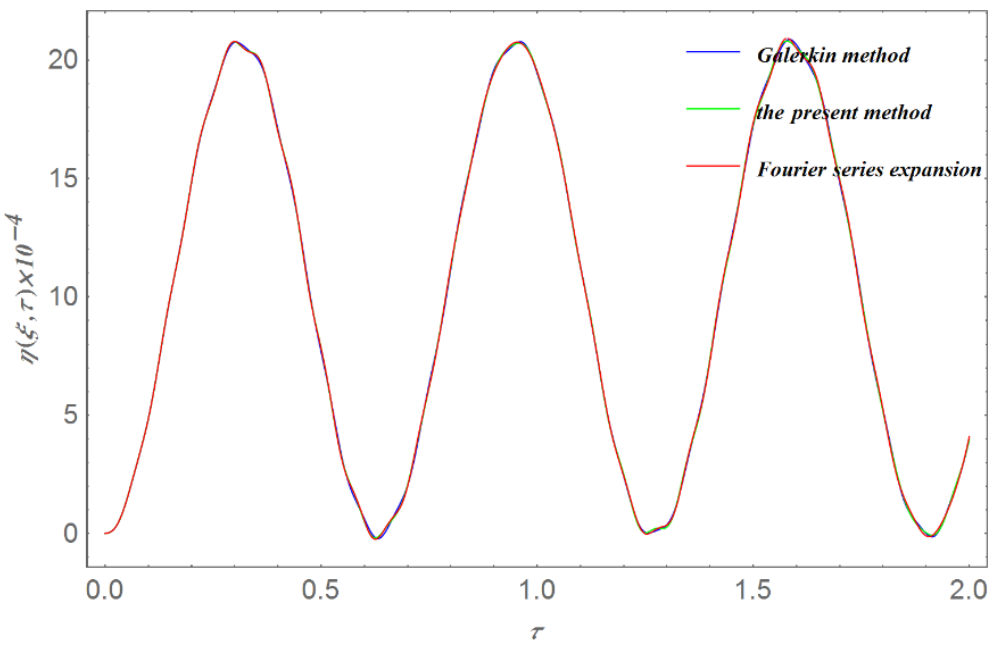

Fig. 3 The S-S time histories of pipe dimensionless displacement at the midpoint $(x=\mathrm{L} / 2)$ using the developed method, Galerkin method and Fourier series expansion technique. 

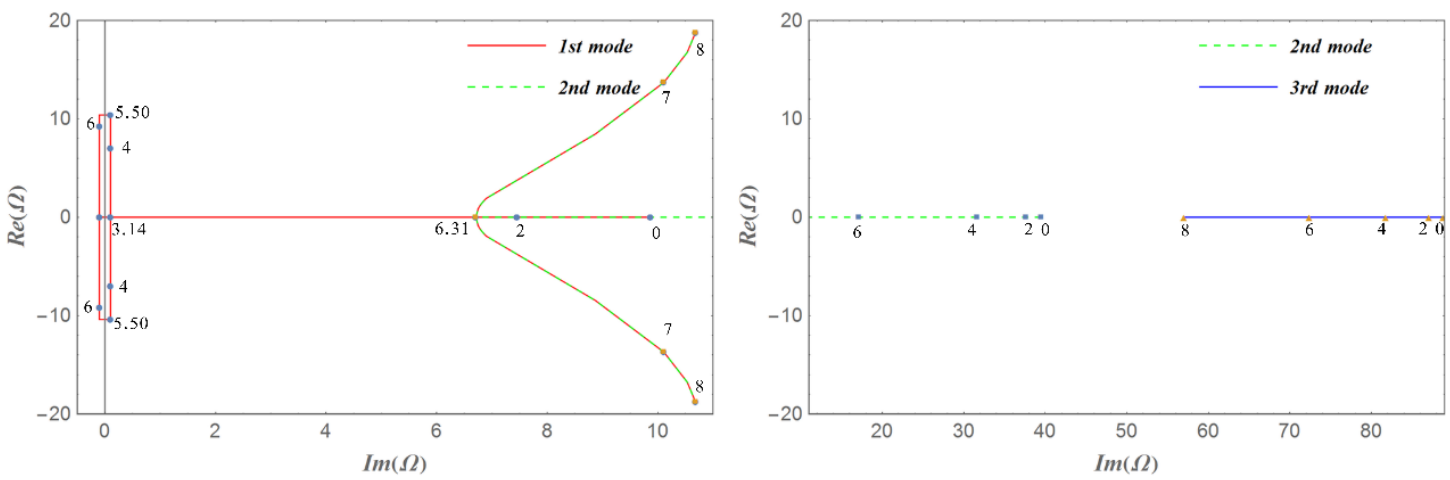

Fig. 4 The first three dimensionless natural frequencies of the S-S fluid-conveying pipe with $\beta=0.5$. 

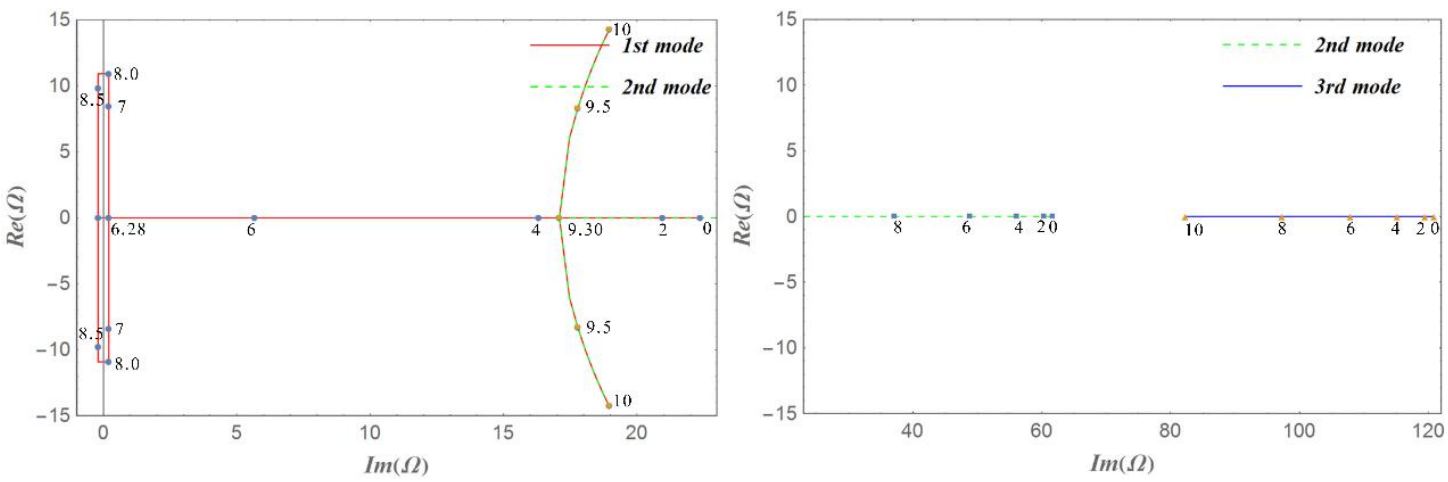

Fig. 5 The first three dimensionless natural frequencies of the $\mathrm{C}-\mathrm{C}$ fluid-conveying pipe with $\beta=0.5$. 

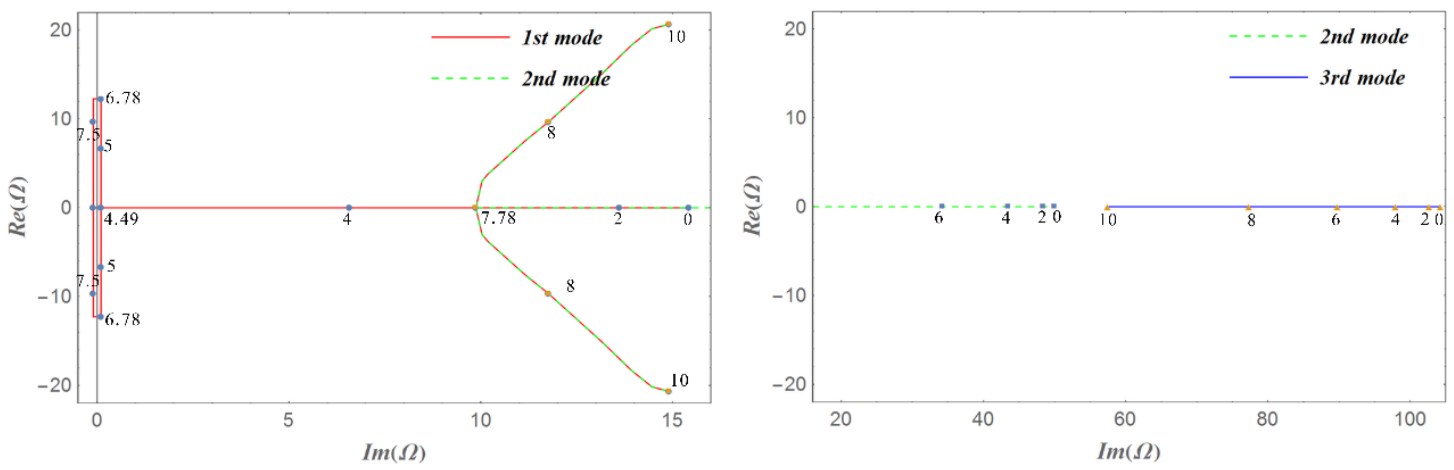

Fig. 6 The first three dimensionless natural frequencies of the $\mathrm{S}-\mathrm{C}$ fluid-conveying pipe with $\beta=0.5$. 


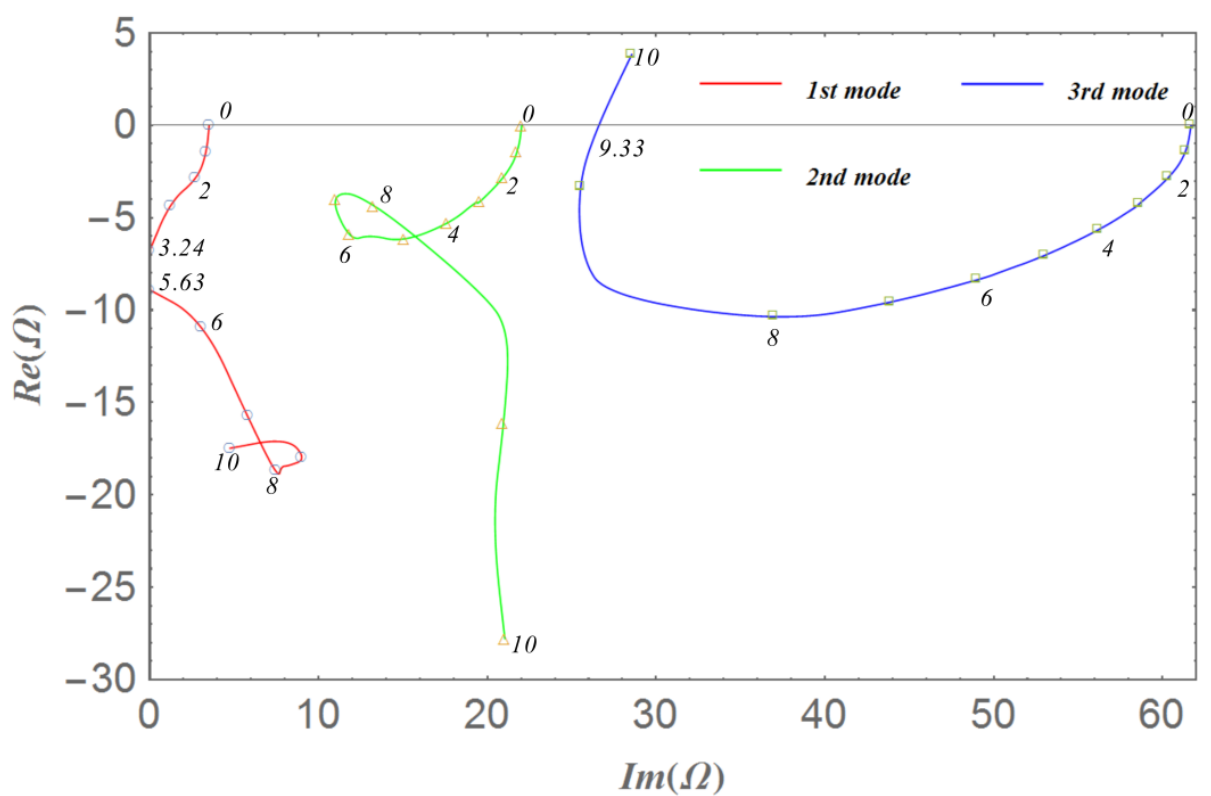

Fig. 7 The first three dimensionless natural frequencies of the $\mathrm{C}-\mathrm{F}$ fluid-conveying pipe with $\beta=0.5$. 


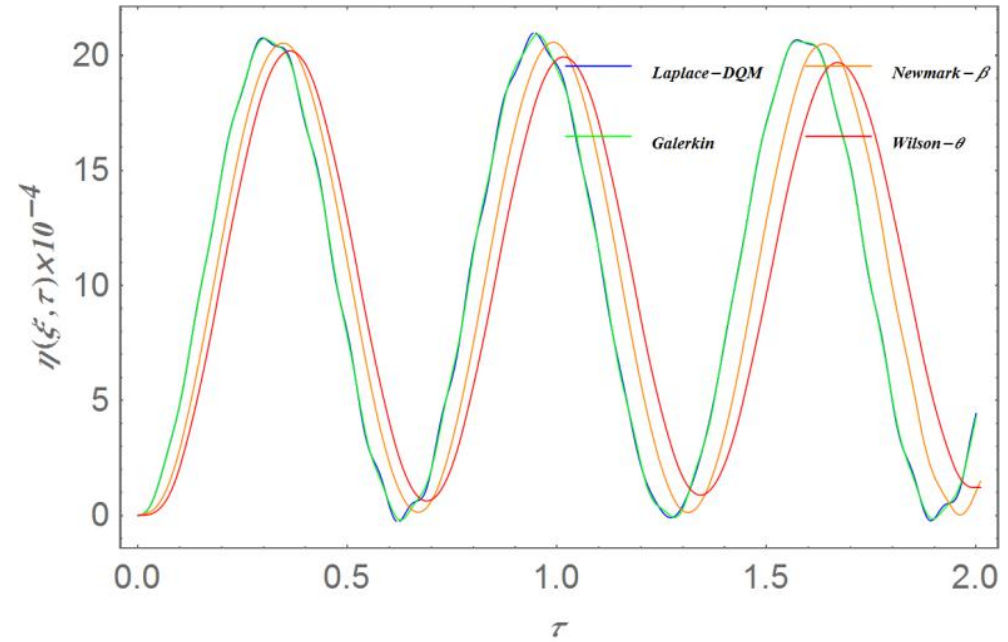

Fig. 8 The $\mathrm{S}-\mathrm{S}$ time histories of the pipe dimensionless displacement at the midpoint $(x=\mathrm{L} / 2)$ using the different methods. 


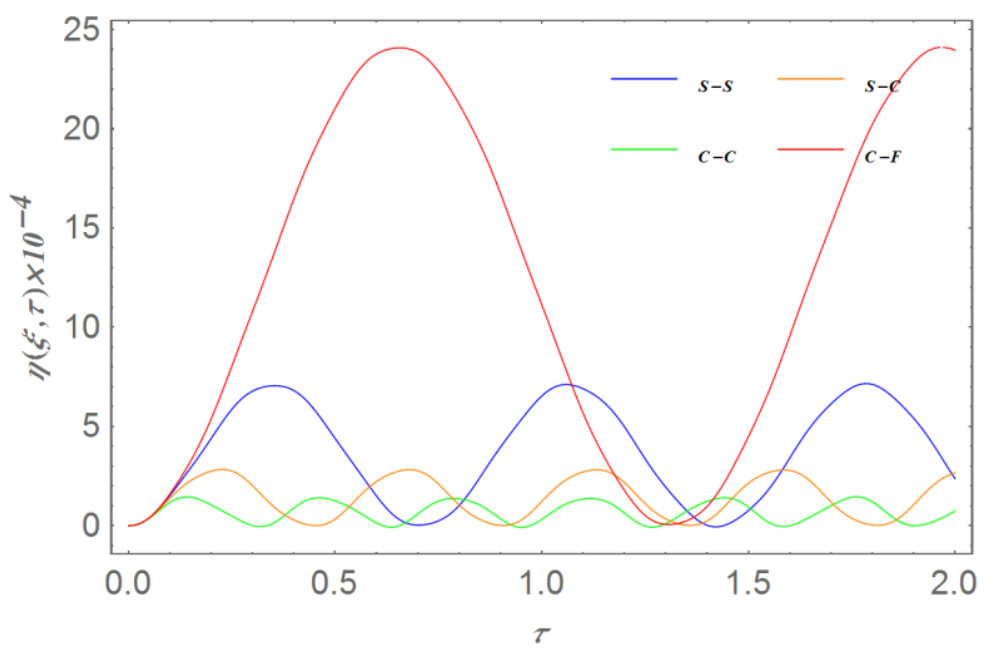

Fig. 9 Time histories of pipe dimensionless displacement under four different sets of end boundary conditions. 


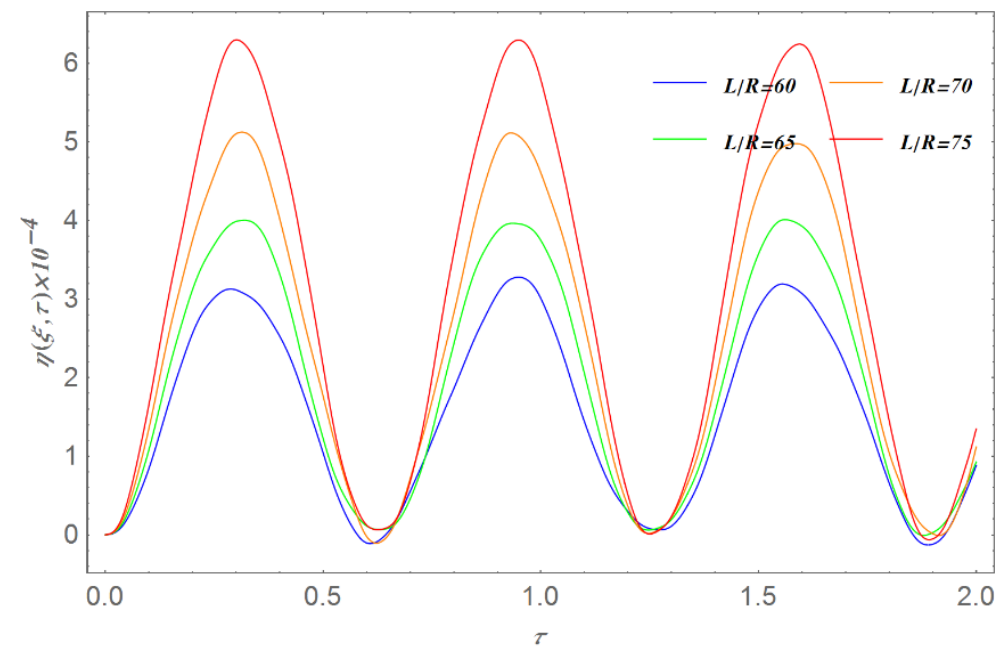

Fig. 10 The S-S time histories of the pipe dimensionless displacement at the midpoint $(x=\mathrm{L} / 2)$ with different length to radius ratios. 


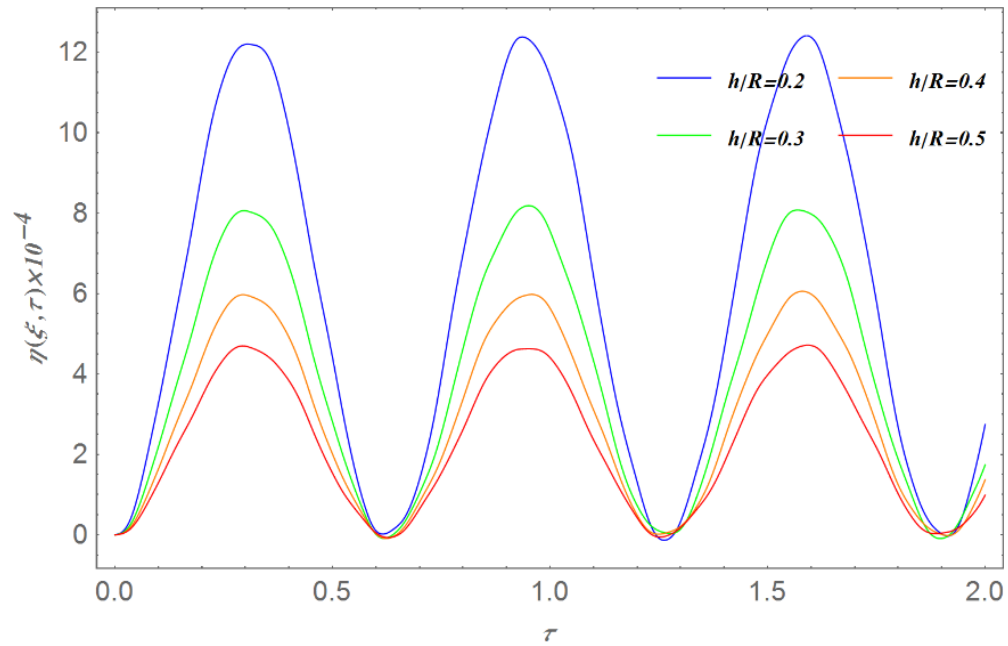

Fig. 11 The S-S time histories of the pipe dimensionless displacement at the midpoint $(x=\mathrm{L} / 2)$ with different thickness to radius ratios. 